\title{
Validation of Test of Nonverbal Intelligence for Pakistani Youth
}

\author{
Muhammad Imtiaz Chaudhry* \\ Shazia Khalid** \\ M. Naeem Mohsin ${ }^{* * *}$
}

\begin{abstract}
The current study was undertaken to establish the validity of new measure of nonverbal intelligence. The test was developed to assess the general cognitive ability of Pakistani youth. The items were constructed keeping in mind to tab the cognitive domains of fluid intelligence, abstract reasoning, spatial ability, working memory, processing speed, inductive and deductive reasoning, application of logics, drawing of inferences and related abilities. Multidimensional construct methods were used to determine the construct validity. The principal factor analysis with varimax rotations yielded a single factor loading, an indicative of uni factor test. Inter correlation of five subscales and total test scores were found to be highly significant $(p<.001)$. The results showed the homogeneity of the test. The discrimination validity of the test was established by correlating the test scores with test takers performance on test of physical agility and stamina. The Pearson correlation produced non significant results between the two measures. The criterion validity was established by correlating the test scores with academic achievements which generated highly significant correlations at $p<.001$. The convergent validity was established by correlation the scores with Raven progressive matrices, verbal intelligence tests and nonverbal intelligence being used at inter services selection boards. The results showed coefficient of correlation .384, .227 and .396 respectively for RSPM, VIT and NVIT which was highly significant at $p .<.001$. The age and grade differences also showed that the mean test score increases with age and grade. The results of the validity studies indicate that the proposed nonverbal test is a valid measure to assess the intelligence of Pakistani youth.
\end{abstract}

Keywords: intelligence, non-verbal, test development, construct validity, criterion validity, convergent validity, discriminant validity

\footnotetext{
${ }_{* *}^{*}$ PhD Scholar, Preston University, Islamabad

${ }^{* *}$ Associate Professor Preston University Islamabad, Pakistan

${ }^{* * *}$ Associate Professor, Government College University Faisalabad Email: mnmohsin71@gmail.com
} 


\section{Introduction}

Intelligence is one of the most discussed topics with myriad implication and utility in the field of human resource induction. Intelligence tests are the indispensible tools for measuring the individual differences in cognitive domains. They give understanding about individual differences and help to place them in jobs according to their potentials (Gottfredson, 2003; Reisberg, 2013).

The vide spread application and popularity give boom to testing movement. In Pakistan many institutions and organization are using intelligence test for personnel selecting and training purposes. Despite the importance of intelligence test in Pakistan there is scarcity of research in test development and no worthwhile efforts are seen in intelligence test development. The majority of intelligence tests being used in Pakistan are adaptation or translations of western tests (Gardezi, 2001; Mehmood, 1991). In the past some efforts are visible but these tests could not get worthwhile recognition being weak in content and methodology and thus laying dormant (Hussain, 2001).

Despite the special appeal and proven utility of intelligence test in personnel selection and placement they are also subject to severe critic due to biases in development and interpretation of results (Gottfredson \& Saklofske, 2009). The issue of culture fairness and confounding of language especially in the foreign origin test resulted in to the development of nonverbal intelligence tests as it is generally believed that nonverbal tests are less likely to favour a particular class, culture or educational background (Goldstein \& Beers 2004).

Validity is essential characteristics in the development of new test. It is related tosoundness or appropriateness of a test or instrument in measuring what it is designed to measure. The degree to which a test or instrument measures what it purports to measure. Validity is the evidence for inferences made about a test score which determine the extent with which the scores are appropriate, meaningful and useful for the intended application of the results. Validity is defined as "the degree to which evidence and theory support the interpretations of test scores for proposed uses of test" (AERA et al., 2014). For a test to be valid it is important that test scores are grounded in the psychological theory and empirical evidence (Furr \& Bacharach, 2014; Sireci \& Sukin, 2013).

The main of aim of present study was to establish the validity of the newly developed test of non verbal intelligence for youth. It is important that a test must be reliable and valid with standardized testing protocols so that the very objective of right person for the right job can be achieved. 


\section{Method}

The objectives of the study were achieved by conducting five different studies. The objectives of the study were to test the construct validity of the test (homogeneity and uni-dimensionality), establish the convergent validity of the test, determine the discriminant validity of the test, evaluate the criterion related validity of the test and, find the grade/ age difference.

\section{Study -I. Construct Validity}

Construct validity of the test provide evidence that test scores correlate with the theoretical concept of the test for which the test is designed to measure. Establishment of construct validity is crucial for the conduct of substantive research and development. The goal of construct validity is to determine whether test scores provide a good measure of specific construct.

\section{Participant}

Sample for the validity studies consists of 400 intermediate level students from different colleges of the country who appeared at inter services selection board Malir for induction into armed forces. The age range of the sample was from 17 to 20 years (avg age 18). The convenient purposive sampling technique was used for data collection.

\section{Procedure}

All the candidates were administered with present test along with specifically designed demographic sheet indicating the information about marks in the last exam, institution name and the medium of instruction. The data was analyzed with the SPSS v23. Multidimensional construct methods were used to determine the construct validity.

\section{Factorial Validity}

Factor analysis is an important method for estimation of construct validity as factors here functions as construct. These factoring provides a good estimate of the test homogeneity. To find the construct validity 
factor principal component analysis with varimax rotation was applied to analyze the dimensionality of test.

\section{Results}

The analysis revealed that test is a uni-factor test as all subtests score do load quite high on this factor which indicates that test is homogeneous and loaded on construct intelligence (Table 1).

Table 1

Principle Component Analysis Showing the Loadings of all Subtest on a Single Factor $\quad(N=400)$

\begin{tabular}{ll}
\hline Category of Subtest & Factor I \\
\hline Matrices & .728 \\
Odds one out & .663 \\
Similarity & .760 \\
Series & .717 \\
Analogies & .768 \\
\hline
\end{tabular}

Table 1 shows the factor loading for matrices, odd one out, similarities, series and analogy items. Results also indicated that all subtests values do load quite high on a single factor which indicates that test is uni factor test for test of fluid intelligence.

Table 2

Total Variance Explained for the test of Nonverbal Intelligence $(N=400)$

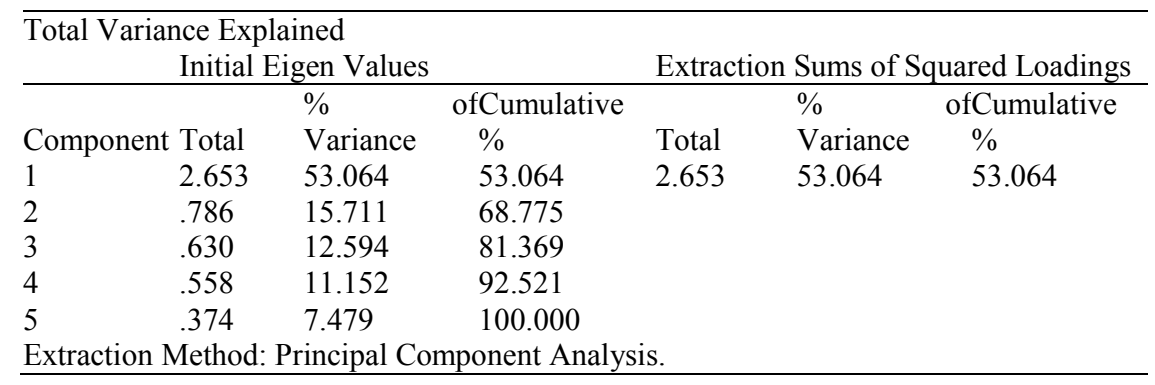




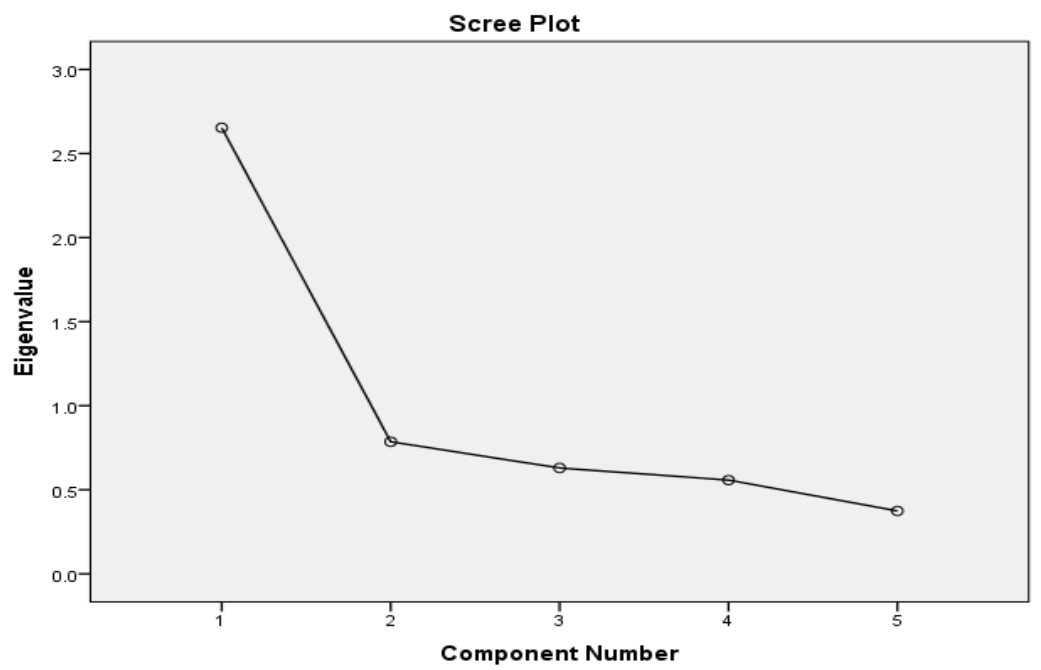

Figure 1: Analysis

\section{Structural Validity (internal consistency)}

Matrix of inter correlations points towards the division of construct into more meaningful part. The relationship between parts indicates toward the homogeneity of the test. To find the consistency among the subtest and full test inter correlation was computed amongst the test and subtest scores by administering the test on the selected sample.

Result yielded high correlation amongst the five subtests and the full test which indicates that the subtests and test are a measure of some single factor i.e., intelligence (Table 3 ).

\section{Table 3}

Index of Inter Subtest and Full Test Correlations amongst the Subtests and the Full Test $(N=400)$

\begin{tabular}{llllll}
\hline Category of Subtest & MAT & ODD & SIM & SER & ANA \\
Matrices & - & & & & \\
Odds one out & $.433^{* * *}$ & & & & \\
Similarity & $.436^{* * *}$ & $.364^{* * *}$ & & & \\
Series & $.438^{* * *}$ & $.454^{* * *}$ & $.410^{* * *}$ & & \\
Analogies & $.403^{* * *}$ & $.307^{* * *}$ & $.465^{* * *}$ & $.467^{* * *}$ & \\
Full Test & $.769^{* * *}$ & $.701^{* * *}$ & $.725^{* * *}$ & $.762^{* * *}$ & $.691^{* *}$ \\
\hline
\end{tabular}

$$
p<.001
$$


Table 3 showed that the inter correlations amongst the test score are highly significant at $P<.001$ which reflects the internal consistency amongst the subtest and full test.

\section{Study II Convergent Validity}

Convergent validity is related to the degree that the test scores are related to other measures that purport to measure the similar construct.

\section{Participant}

Sample for the validity studies consists of 400 selected intermediate level students from different colleges of the country who appeared at inter services selection board Malir for selection in armed forces. The age range of the sample was from 17 to 20 years (avg age 18).

\section{Instruments}

\section{Raven Standard Progressive Matrices (RSPM)}

Ravens Progressive Matrices (Ravens, Court, \& Ravens, 1978) is a standard non-verbal test of intelligence designed to measure a systematic reasoning consisted of 60 problems which are further divided into five sets of 12 questions each with increasing difficulty levels. High score indicates high non-verbal cognitive ability and vice versa. Raven Progressive Matrices (RPM) is widely used test to assess the cognitive abilities of the test takers.

\section{Verbal intelligence test (VIT)}

This test is indigenously developed by Psychological Research Wing of Personnel Selection Directorate to assess the cognitive level of candidates. The test consists of 80 verbal items including, verbal series, analogies, and reasoning items. The items of arithmetic reasoning and vocabulary are also included in the tests. The time limit for the test is 30 minutes.

\section{Non Verbal intelligence test (NVIT)}

This test is the adaptive version of Matrices which is designed to assess the cognitive function of candidates at Inter Services and selection Boards. The test consists of 64 matrices with relative increasing difficulty level. The time limit for the test is 30 minutes. 


\section{Procedure}

To establish the convergent validity proposed test was administered on the selected sample of 400 candidates along with Raven Standard Progressive Matrices (RSPM), Verbal Intelligence Test (VIT) and Non Verbal Intelligence Test (NVIT) applied in ISSB for intelligence testing. The candidates were also administered a specifically designed demographic sheet indicating the information about marks in the last exam, institution name and the medium of instruction. The scores were then correlated with the scores of other measures i.e., RPM, VIT and NVIT.

The significant high correlation with RSPM VIT and NVIT at is an indicator of convergent validity (Table 4).

Table 4

Correlations Coefficient of RSPM, VIT and NVIT with the Subtest and the Full Test $\quad(N=400)$

\begin{tabular}{llll}
\hline \multirow{2}{*}{ Category of Subtest } & \multicolumn{2}{l}{ Convergent validity } & \\
& RSPM & VIT & NVIT \\
\hline Matrices & $.234^{* * *}$ & $.221^{* * *}$ & $.225^{* * *}$ \\
Odds one out & $.215^{* * *}$ & $.219^{* * *}$ & $.241^{* * *}$ \\
Similarity & $.335^{* * *}$ & $.165^{* *}$ & $.359^{* * *}$ \\
Series & $.362^{* * *}$ & $.109^{*}$ & $.368^{* * *}$ \\
Analogies & $.293^{* * *}$ & $.117^{*}$ & $.262^{* * *}$ \\
Full Test & $.384^{* * *}$ & $.227^{* * *}$ & $.396^{* * *}$ \\
\hline
\end{tabular}

$* \mathrm{p}<.05$

Table 4 showed that all subtest and full test has significant correlation with RSPM, VIT and NVIT tests.

\section{Study III Discriminate Validity}

Discriminate validity is related to the evidence that the test score are not related to other measures that are designed to measure different and often opposite construct. To establish the discriminate validity proposed test results were correlated with the test score of Individual Obstacles (a test of physical agility and stamina). 


\section{Participants}

Sample for the validity studies consists of 400 intermediate level students from different colleges of the country who appeared at inter services selection boards for intelligence and personality testing. The age range of the sample was from 17 to 20 years (avg age 18).

\section{Instruments}

\section{Individual obstacles}

This is a performance test designed to assess the stamina and physical agility of the candidates at inter services selection boards. The test consists of a set of nine individual obstacles for the time limit is two minutes.

\section{Procedure}

To find the discriminant validity of the test the proposed test was administered on the selected sample of candidates at the Inter Services Selection Board Malir. To find the relationship between two measures (tests scores and points on individual obstacles) the Pearson product moment correlation formula was applied to get the correlation coefficient.

The results showed a non significant relationship between the test scores and the points on the individual obstacles (Table 5).

Table 5

Correlations Coefficient of Subtests and the Full Test Score with Individual Obstacles $(N=400)$

\begin{tabular}{ll}
\hline Category of Subtest & $\begin{array}{l}\text { Discriminant Validity } \\
\text { Individual Obstacles }\end{array}$ \\
\hline Matrices & $-.018 \mathrm{~ns}$ \\
Odds one out & $.036 \mathrm{~ns}$ \\
Similarity & $.101 \mathrm{~ns}$ \\
Series & $-.004 \mathrm{~ns}$ \\
Analogies & $.007 \mathrm{~ns}$ \\
Full Test & $.044 \mathrm{~ns}$ \\
\hline
\end{tabular}

Table 5 shows that all subtest and full test has no significant correlation with Individual Obstacles (IOs). 


\section{Study IV Criterion-Related Validity}

Criterion validity is related to the degree to which test's scores correlate with the score of the other measureable and relevant variable.

\section{Participants}

Sample for the validity studies consists of 400 intermediate level students from different colleges of the country who appeared at inter services selection boards for intelligence and personality testing. The age range of the sample was from 17 to 20 years (avg age 18).

\section{Instrument}

\section{Academic achievement}

The academic achievement was measured by the percentages of scores achieved by candidates in the last examinations.

\section{Procedures}

To determine the criterion validity proposed test was administered on the selected sample of 400 students and then the test scores were correlated with the percentages of their marks in the intermediate level and equivalent examinations.

The results show a significant positive relationship between the subtest scores and the percentage of marks obtained by students in their last examination (Table 6).

Table 6

Correlation between Test Scores and the Marks in the Last Examinations $(N=400)$

\begin{tabular}{ll}
\hline Category of Subtest & Coefficient of criterion validity \\
\hline Matrices & $.243^{* * *}$ \\
Odds one out & $.231^{* * *}$ \\
Similarity & $.191^{* *}$ \\
Series & $.213^{* * *}$ \\
Analogies & $.198^{* * *}$ \\
Full Test & $.321^{* * *}$ \\
\hline
\end{tabular}

$* P<.05$ 
Table 6 shows that there is significant correlation with subtest and full test score and the marks in last exam.

\section{Grade / Age Differences}

A major criterion used for the validation of intelligence test is age differences. The intelligence is expected to increase with the grade and age progression. As cognitive abilities are expected to improve with increase in grade and age therefore it is appropriate to administer the present test on students of different grades and age limits. To establish the grade and age differences a separate study was conducted on sample of school and college level students.

\section{Study V Grade Differences}

\section{Participant}

Sample of the study consists of 300 students of Army public School and College students of Malir Cantonment Karachi. 100 students were of grade of $10^{\text {th }}$ grade (Avg age 15.2), 100 students of $11^{\text {th }}$ grade (Avg age 16.4 ) and 100 students of $12^{\text {th }}$ grader (Avg age 17.8). Convenient random sampling method was used for data collection.

\section{Procedure}

To establish the grade and age validity test was administered by the researcher on the 300 selected students of Army public School and College students of Malir Cantonment Karachi. For each grade level 100 students were selected. The data was put through analysis and mean scores and standard deviations were computed for each grade and age levels. The mean values were compared to find the score differences amongst the three grad and age groups.

Results show mean differences amongst the three group scores. Significant differences were found amongst the three groups which indicate that the three groups are different from each other in their test performance. The data shows that the mean score for 12 grade students are higher than the mean of 11 and 10 grade students for the subtest and the full test for the test of nonverbal intelligence. The average score for age levels also showed the similar results for different age groups on the subtest and the full test (Table 7). 
Table 7

Grade Wise and Age Wise Differences on the Test of Nonverbal Intelligence Test $(N=300)$

\begin{tabular}{lllllll}
\hline $\begin{array}{l}\text { Nonverbal } \\
\text { test/ subtest }\end{array}$ & \multicolumn{2}{l}{$\begin{array}{l}\text { Grade10 } \\
\text { (15 years 4 month) }\end{array}$} & \multicolumn{2}{l}{$\begin{array}{l}\text { Grade11 } \\
\text { (16 years 6 month) }\end{array}$} & \multicolumn{2}{l}{$\begin{array}{l}\text { Grade12 } \\
\text { (17 years 8 month) }\end{array}$} \\
\hline & $M$ & $S D$ & $M$ & $S D$ & $M$ & $S D$ \\
Matrices & 9.22 & 2.865 & 10.02 & 2.885 & 11.42 & 3.125 \\
Odds & 6.53 & 2.123 & 7.14 & 2.234 & 7.40 & 2.562 \\
Similarity & 6.61 & 2.387 & 7.97 & 2.561 & 8.46 & 2.787 \\
Series & 7.83 & 2.423 & 8.63 & 2.411 & 9.10 & 2.583 \\
Analogies & 6.60 & 2.012 & 8.08 & 2.114 & 8.70 & 2.132 \\
Full Test & 37.68 & 8.670 & 40.08 & 9.166 & 45.08 & 9.670 \\
\hline
\end{tabular}

Table 7 shows that the there is significant progressive increase in the mean scores for the three group. Results indicate that mean scores for the three groups increase with increase in the grade and age group which is indicative of grade and age validity.

\section{Discussion}

The main aim of this part of study was the establishment of validity of the test of nonverbal intelligence. In the evaluation of the quality of any psychological measure validity is very important as it is related to accuracy of interpretation and use of the test. Different Validity studies were conducted to determine the ability of the test to measure the intelligence. To find the construct validity factor analysis, convergent and divergent validity methods were applied. Construct validity of the test was determined by factor analysis method and inter correlations amongst the subtest and the full test scores. Data shows the factorial validity of the test on a single factor perceived as the measure of nonverbal intelligence. The varimax principal factor analysis reflects a factor loading of $.728, .663,760, .717$, and .768 for matrices, odd one out, similarities, series and analogy items respectively. Findings show that all subtests values do load quite high on a single factor which indicates that test is uni factor test for test of nonverbal intelligence.

To find the structural validity consistency amongst subtest and the full test was computed. The correlation coefficient values ranges from .307 to .369 . The matrix of correlation shows a significant highly positive correlation at $p<.001$ amongst the subtest and the full test. The correlation coefficient reflects the internal consistency amongst the subtest and full test. The significant positive correlation amongst all the 
indices indicated that the test is taping some universal factor as general factor which is congruence to the theoretical foundations of the study.

To establish the convergent validity test scores were correlated with the scores of other measures that purport to measure the similar construct. Raven Standard Progressive Matrices (RSPM), Verbal Intelligence Test (VIT) and Non Verbal Intelligence Test (NVIT) were selected for comparison. The correlation between all subtest and full test has significant correlation with RSPM, ranges from .215 to .362 significant at $p<.005$ for the subtests and .384 significant $p<.001$ for the full test. Similarly significant correlation of subtest and full test was observed with the VIT which ranges from .109 to .221 significant at $p<$ .05 for the subtests and .227 significant at $p<.001$ for the full test. The correlation between NVIT and subtest and the full test ranges from .215 to .362 for the subtests and .384 for the full test which are significant at $p$ $<.001$. The significant high correlation with RSPM, VIT and NVIT at is an indicator of convergent validity. The significant correlation can be cited as the evidence that new test measure the same ability area that has been measured by established tests (Anastasi, 1997).

The discrimination validity of the test was determined by correlating the test scores with some other measure not related to intelligence. The scores of the test were correlated with another measure of physical strength and stamina, Individual Obstacles (IOs). The results show a no significant results which indicates that the two measures are not related. Low correlation coefficient between two measures of different traits provides evidence of divergent validity (Furr, 2014).

Criterion validity of the test was established by correlating the test scores with marks in last exam. The correlation with Matrices was .243 significant at $p<.001$, odd one out .231 significant at $p<.001$, similarity .191 significant at $p<.005$, series .213 significant at $p<.001$ and analogies .199 significant at $p<.001$. The correlation with full test and marks in the last exam was .321 significant at $p<.001$. The results show a significant high correlation with subtest and full test score and the marks in last exams. Significantly high correlation indicates that test has criterion related (concurrent) validity.

Eysenck (2001) found that intelligence has strong relationship with academic achievement and future success in different fields of life. Therefore intelligence test can be used as predictor of future success. Other studies also give evidence of causal relationship with intelligence and academic achievements. Intelligence test provides an objective measure of individual differences in intellectual and cognitive domains. Gottfredson found that fluid intelligence is a good predictor of 
performance in school, university, and cognitively demanding occupations (Gottfredson, 1998; Jensen, 1998).

The concurrent intelligence and academic performance is one of the primary methods of diagnosing the learning disabilities (Yen, Konold, \& Mcdermott, 2004). Review of various authors reach at conclusion that relationship of cognitive tests with education correlates ranging from 0.40 to 0.63 (Barlets, Rietveld, Van Vall, \& Boomsma, 2002b; Brody, 1992; Jensen, 1998; Neisser et al., 1996; Sternberg, Grigorenko, \& Bundy, 2001; Sambo, 2014).

To age and grade validity is also considered an important parameter of validity studies. The grade and age validity is especially important for construct which are assumed to increase with the age and advancement educational grades and achievement. The findings of the study shows a successive increase in the mean score of the three grade and age groups. The increase in mean score with developing age and higher grades suggest that test has grade and age validity.

\section{Conclusion}

The validity analysis of the test reflects the test is a homogeneous and loaded on a single factor of intelligence. The test is good predictor of future success in the field of educations as the test scores are highly correlated with marks in the last exam. Overall test is psychometrically sound measure for the assessment of intellectual capability of the test takers. 


\section{References}

AERA, APA, \& NCME. (1999). Standards for educational and psychological testing. Washington DC: American Educational Research Association.

Anastasi, A., \& Urbina, S. (1997). Psychological Testing. (7th ed.), Upper Saddle River, NJ: Prentice Hall.

Bartels, M., \& Van Baal, G. (2002). Heritability of educational achievement in 12-year-olds and the overlap with cognitive ability. Twin Research, 5, 544-553.

Furr, R. M. \& Bacharach, V. R. (2014). Psychometrics: an introduction $\left(2^{\text {nd }}\right.$ ed $)$. Loss Angeles: Sage publication.

Gardezi, A. H. (2001). Development and standardization of an indigenous non-verbal test for adolescents (Unpublished Doctoral dissertation). National Institute of Psychology, Quaid-i-Azam University, Islamabad, Pakistan.

Gottfredson, L. S. (1998). The general intelligence factor. Scientific American Presents, 9(4), 24-29.

Gottfredson, L., (2003). Dissecting practical intelligence theory: Its claims and its evidence. Intelligence, 31, 343-397.

Gottfredson, L. \& Saklofske, D. H. (2009). Intelligence: Foundations and issues in assessment. Canadian Psychology, 50(3), 183-195. doi: $10.1037 / \mathrm{a} 0016641$

Hussain, S. S. (2001). Development, validation, and standardization of a group verbal intelligence test in Urdu for adolescents (Unpublished Doctoral dissertation). National Institute of Psychology, QuaidiAzam University, Islamabad, Pakistan.

Jensen, A. R. (1998). The g factor. The science of mental ability. Westport, Connecticut: Praeger Publishers.

Mahmood, Z. (1991). Intelligence, IQ and the third world. Pakistan Journal of Psychological Research, 6 (1-2), 31-53.

Neisser, U., Boodoo, G., Bouchard, T. J., Jr., Boykin, A. W., Brody, N., Ceci, S., \& Urbina, S. (1996). Intelligence: Knowns and unknowns. American Psychologist, 51(2), 77-101. 
Raven, J., Raven, J. C., \& Court, J. H. (1978). Standard matrices: Manual for raven's progressive matrices and vocabulary scales. London:

Reisberg, D. (2013). The Oxford handbook of cognitive psychology. New York, NY, US: Oxford University Press. Retrieved from: http://dx.doi.org/10.1016/j.intell.2017.06.001

Sambo, A. (2014). Relationship of non-verbal intelligence materials as catalyst for academic achievement and peaceful co-existence among Secondary School students in Nigeria. Journal of Education and Practice, 6(31),62-66.

Sireci, SG., \& Sukin, T. (2013). Test Validity APA Handbook of testing and assessment in psychology. Washington, DC: American Educational Research Association.

Sternberg, R. J., Grigorenko, E. L., \& Bundy, D. A. (2001). The predictive value of IQ. Merrill-Palmer Quarterly, 47, 1-41. doi:10.1353/mpq.2001.0005

Yen, C.-J., Konold, T. R., \& McDermott, P. A. (2004). Does learning behavior augment cognitive ability as an indicator of academic achievement? Journal of School Psychology, 42(2), 157169.doi.org/10.1016/j.jsp.2003.12.001

\section{Citation of this Article:}

Chaudhry, M. I., Khalid, S., \& Mohsin, M. N. (2018). Validation of test of non-verbal intelligence for Pakistani youth. Pakistan Journal of Education, 35(2), 223-237.

Received on: January

05,2018

Revised on: July

06,2018

Accepted on: July

26, 2018 